\title{
Computer Graphics and a New Gibbs Phenomenon for Fourier-Bessel Series
}

Alfred Gray and Mark A. Pinsky

\section{CONTENTS}

1. Introduction

2. Analysis of the Expansion in $J_{0}$

3. Analysis of the Expansion in $\mathrm{J}_{\mathrm{m}}$, with $\mathrm{m}>0$

Note on the plots

References

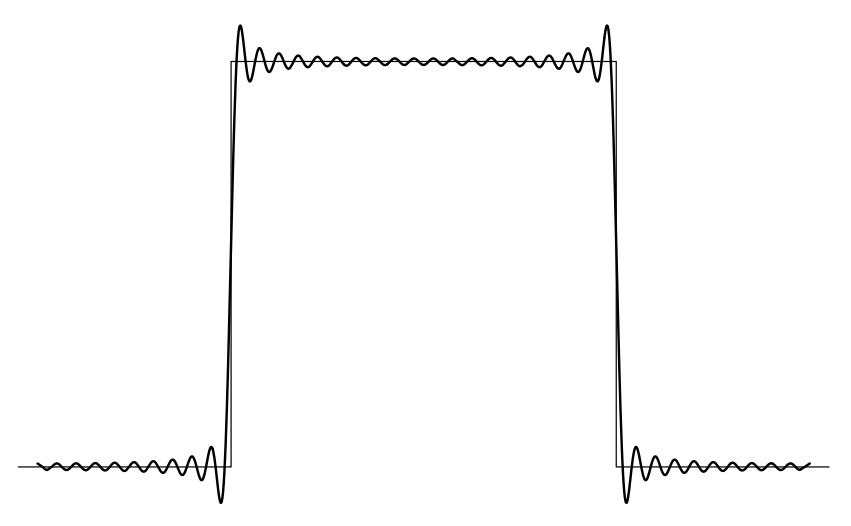

We report the existence of a Gibbs-like phenomenon at points of continuity in the expansion of functions in Fourier-Bessel series.

\section{INTRODUCTION}

It is well known that many functions can be expanded in Fourier-Bessel series. (A Fourier-Bessel series is one of the form

$$
\sum_{k=1}^{\infty} a_{k} J_{m}\left(x x_{k}^{(m)}\right)
$$

where $x_{k}^{(m)}$ denotes the $k$-th positive zero of the Bessel function $J_{m}$.) It is not surprising that partial sums of the Fourier-Bessel series of a piecewise continuous function have an overshoot at the points of discontinuity; this behavior is familiar from partial sums of ordinary Fourier series, as in the figure on the left, and is called the Gibbs phenomenon [Gibbs 1898; Weyl 1909].

Let's compare the graphs of the partial sums of the Fourier and Fourier-Bessel expansions of the function $f(x)=1$ for the interval $-1<x<1$. The expansions are

$$
\frac{1}{2}+\sum_{k=1}^{\infty} \frac{2(-1)^{k+1}}{(2 k-1) \pi} \cos \frac{(2 k-1) \pi x}{2}
$$

and

$$
2 \sum_{k=1}^{\infty} \frac{J_{0}\left(x x_{k}^{(0)}\right)}{x_{k}^{(0)} J_{1}\left(x_{k}^{(0)}\right)}
$$

where we took $m=0$ in the Fourier-Bessel expansion (we will consider other values of $m$ later). A partial sum (up to $k=20$ ) for the ordinary Fourier series is what is shown on the left; the graph for 
the Fourier-Bessel series is shown below, in Figure 1. Both series converge to 1 on the interval $-1<x<1$. At $x= \pm 1$ they vanish, so we expect the Gibbs phenomenon at these points, and indeed we observe it in the graphs.

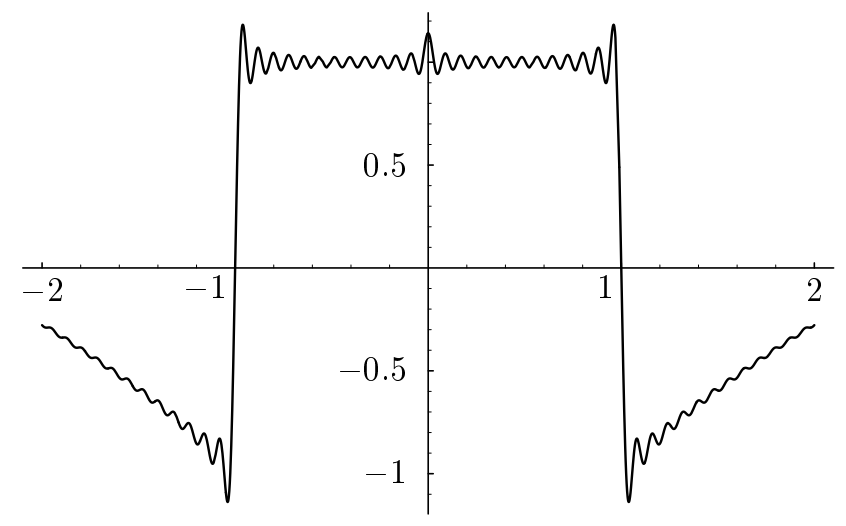

FIGURE 1. The graph of $2 \sum_{k=1}^{20} \frac{J_{0}\left(x x_{k}^{(0)}\right)}{x_{k}^{(0)} J_{1}\left(x_{k}^{(0)}\right)}$

What is intriguing about the plot of the FourierBessel series is that there also seems to be a Gibbslike phenomenon at $x=0$, which is a point of continuity. We shall see in Section 2 that this is due to the fact that the series converges more slowly at $x=0$ than at surrounding points.

The first author observed this unusual behavior when he was using Mathematica to draw graphs for the appendix to [Pinsky 1991]. Before the advent of computer programs such as Mathematica, graphs of partial sums of Fourier-Bessel series were difficult to obtain. That Wilbraham published the first graphs of the ordinary Gibbs phenomenon in 1848 is quite remarkable [Hewitt and Hewitt 1980; Wilbraham 1848]. The Gibbs-like phenomenon described here is a good example of how computer graphics can suggest new mathematical results.

The ordinary Gibbs phenomenon for Bessel functions was investigated analytically by Cooke [1928], who showed that it is not present at $x=0$. Cooke did not observe that a slower rate of convergence is possible.

A natural generalization of the expansion in $J_{0}$ just discussed is the expansion

$$
2 \sum_{k=1}^{\infty} \frac{J_{m}\left(x x_{k}^{(m)}\right)}{x^{m} J_{m+1}\left(x_{k}^{(m)}\right) x_{k}^{(m)}}
$$

for $f(x)=1$ in $-1<x<1$ [Pinsky 1991, p. 192]. Although $m$ may be any real number with $m>-1$, we shall limit ourselves to a discussion of the case when $m$ is an integer. If we were to plot (1.1), we would run into practical difficulties, since the sums become unbounded near $x=0$ if $m>\frac{1}{2}$. In order to overcome this difficulty, we work instead with the associated convergent expansion

$$
2 \sum_{k=1}^{\infty} \frac{J_{m}\left(x x_{k}^{(m)}\right)}{J_{m+1}\left(x_{k}^{(m)}\right) x_{k}^{(m)}}
$$

for $x^{m}$ in $-1<x<1$. Figure 2 illustrates the slower convergence near $x=0$ in the cases $m=$ $1,2,3,4$. The theoretical analysis is given in Section 3 .

\section{ANALYSIS OF THE EXPANSION IN J}

Let $f(x)$ be a function for which an expansion

$$
f(x)=\sum_{k=1}^{\infty} a_{k} J_{m}\left(x_{k}^{(m)} x\right)
$$

is valid on the interval $-1<x<1$. It follows from standard properties of Bessel functions (see [Watson 1966, Chap. XVIII], for example) that the coefficients $a_{k}$ are given by

$$
a_{k}=\frac{2}{J_{m+1}\left(x_{k}^{(m)}\right)^{2}} \int_{0}^{1} t f(t) J_{m}\left(x_{k}^{(m)} t\right) d t .
$$

We will use the following asymptotic expansions [Abramowitz and Stegun 1965, pp. 371, 364], both of which hold for fixed $m$ :

$$
J_{m}(x)=\sqrt{\frac{2}{\pi x}}\left(\cos \left(x-\frac{(2 m+1) \pi}{4}\right)+O\left(\frac{1}{x}\right)\right)
$$

as $x \rightarrow \infty$, and

$$
x_{k}^{(m)}=\left(k+\frac{2 m-1}{4}\right) \pi+O\left(\frac{1}{k}\right)
$$

as $k \rightarrow \infty$. From these relations it is elementary to prove that the asymptotic behavior of

$$
\frac{J_{0}\left(x x_{k}^{(0)}\right)}{x_{k}^{(0)} J_{1}\left(x_{k}^{(0)}\right)}
$$

as $k \rightarrow \infty$ is

$$
\frac{(-1)^{k-1} \cos \left(\frac{1}{4} \pi(1+x-4 k x)\right)}{\left(k-\frac{1}{4}\right) \pi \sqrt{x}}+O\left(\frac{1}{k^{2}}\right)
$$




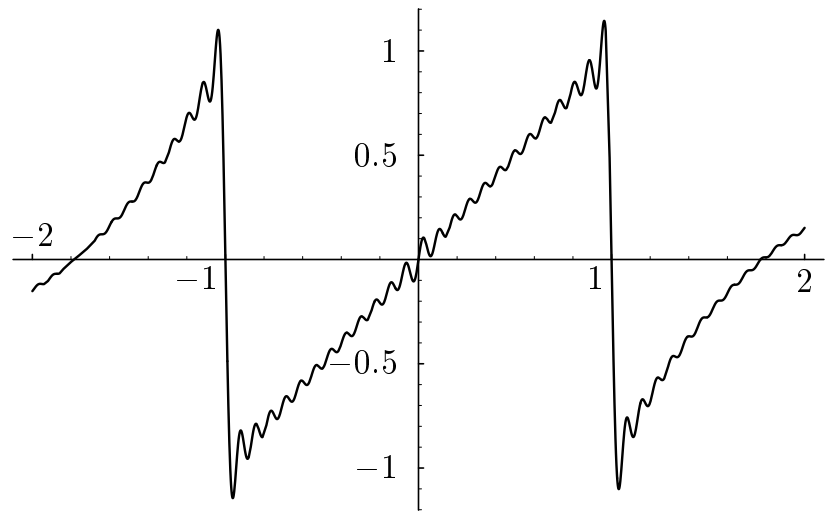

$m=1$

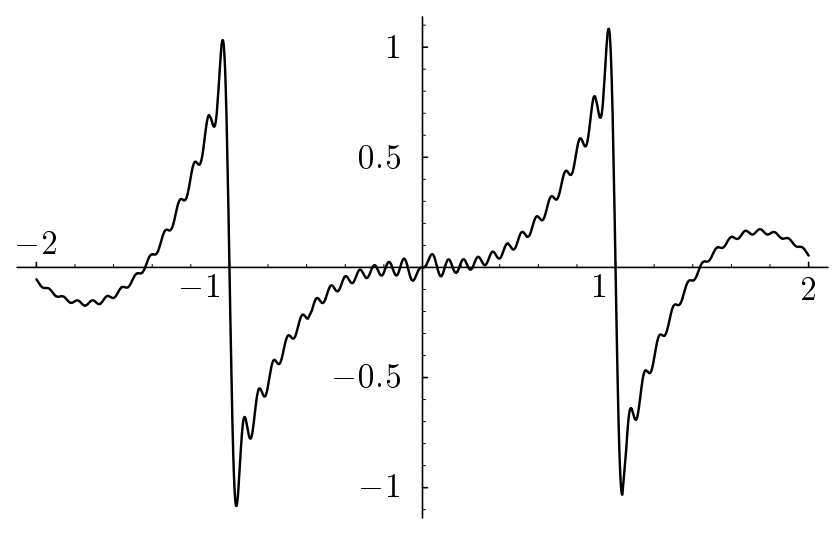

$m=3$

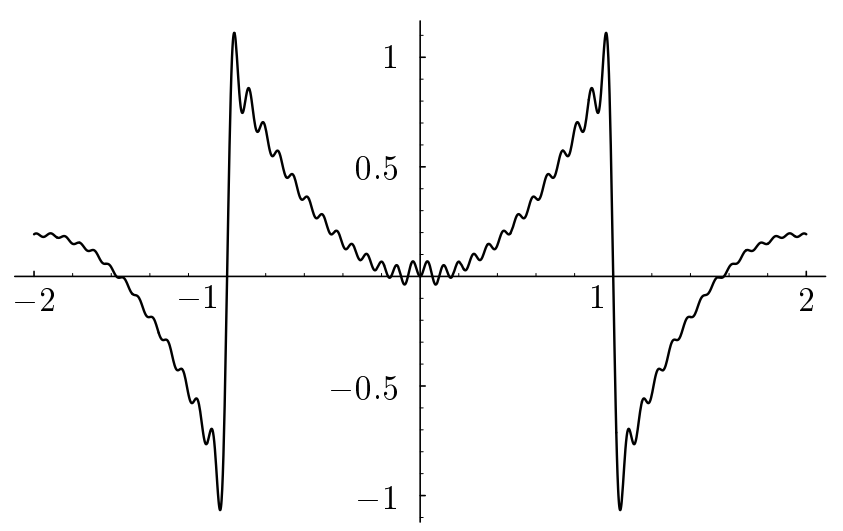

$m=2$

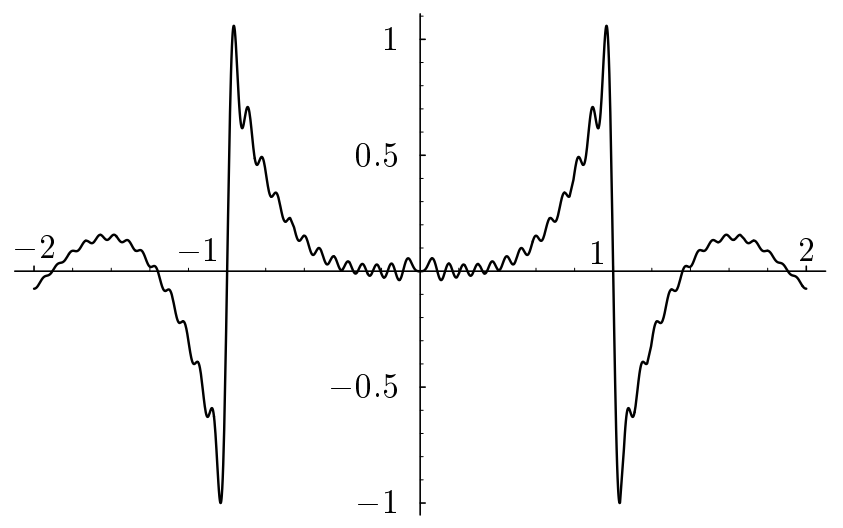

$m=4$

FIGURE 2. Partial sums of the $m$-th order Fourier-Bessel series $2 \sum_{k=1}^{25} \frac{J_{m}\left(x x_{k}^{(m)}\right)}{J_{m+1}\left(x_{k}^{(m)}\right) x_{k}^{(m)}}$, which approximate $x^{m}$
in the interval $-1<x<1$.

for $x \neq 0$, but for $x=0$ it is

$$
\frac{(-1)^{k-1} \sqrt{2}}{\sqrt{4 k-1}}+O\left(\frac{1}{k^{3 / 2}}\right) .
$$

Therefore, as mentioned above, the Gibbs-like phenomenon is caused by the slower rate of convergence at $x=0$ than at surrounding points. More precisely:

Theorem 1. The order-zero Fourier-Bessel expansion for $f(x)=1$ in the interval $-1<x<1$ has the rate of convergence of the series

$$
\sum_{k=1}^{\infty} \frac{(-1)^{k-1} \cos \left(\frac{1}{4} \pi(1+x-4 k x)\right)}{\left(k-\frac{1}{4}\right) \pi \sqrt{x}}
$$

for $x \neq 0$, but for $x=0$ it has the slower rate of convergence of the series

$$
\sum_{k=1}^{\infty} \frac{(-1)^{k-1} \sqrt{2}}{\sqrt{4 k-1}}
$$

\section{ANALYSIS OF THE EXPANSION IN $J_{m}$, WITH $m>0$}

We now consider the expansion

$$
2 \sum_{k=1}^{\infty} \frac{J_{m}\left(x x_{k}^{(m)}\right)}{x^{m} J_{m+1}\left(x_{k}^{(m)}\right) x_{k}^{(m)}}
$$

for the constant function $f(x)=1$ in the interval $-1<x<1$. At $x=0$ the terms of (3.1) are defined by continuity, using the relation 


$$
\lim _{x \rightarrow 0} \frac{J_{m}(a x)}{x^{m}}=\frac{a^{m}}{2^{m} m !},
$$

so the series takes the form

$$
2 \sum_{k=1}^{\infty} \frac{\left(x_{k}^{(m)}\right)^{m-1}}{J_{m+1}\left(x_{k}^{(m)}\right)}
$$

By (2.1) and (2.2) we have

$$
\frac{\left(x_{k}^{(m)}\right)^{m-1}}{J_{m+1}\left(x_{k}^{(m)}\right)}=(-1)^{k} \frac{k^{m-\frac{1}{2}}}{2^{m} m !}\left(1+O\left(\frac{1}{k}\right)\right)
$$

as $k \rightarrow \infty$. On the other hand, for $x \neq 0$ we have

$$
\frac{J_{m}\left(x x_{k}^{(m)}\right)}{x^{m} J_{m+1}\left(x_{k}^{(m)}\right) x_{k}^{(m)}}=\frac{(-1)^{k}}{k x^{m} 2^{m} m !}\left(1+O\left(\frac{1}{k}\right)\right) \text {. }
$$

To summarize:

Theorem 2. The terms of the m-th order FourierBessel series for the function $f(x)=1$ in the interval $-1<x<1$ are asymptotically equivalent to the terms of the convergent series

$$
\frac{1}{2^{m} m ! x^{m}} \sum_{k=1}^{\infty} \frac{(-1)^{k}}{k}
$$

when $x \neq 0$, and to the terms of the series

$$
\frac{1}{2^{m} m ! x^{m}} \sum_{k=1}^{\infty}(-1)^{k} k^{m-\frac{1}{2}}
$$

(which diverges for $m \geq \frac{1}{2}$ ) when $x=0$.

\section{NOTE ON THE PLOTS}

All figures were generated using Mathematica. The requisite values of $x_{k}^{(m)}$ (see (1.1), for instance) were tabulated for each $k \leq 20$ and each $m$; for higher values of $k$ the asymptotic formula (2.2) is good enough, at least for small $m$. Due to the nature of the functions, the PlotPoints option to Plot had to be explicitly set to a relatively high value - around 200 - or some oscillations would be missed. (PlotPoints controls the fineness of the initial subdivision of the domain; after that, an adaptive algorithm takes over.)

\section{REFERENCES}

[Abramowitz and Stegun 1965] M. Abramowitz and I. A. Stegun, Handbook of Mathematical Functions, Dover, New York, 1965.

[Cooke 1928] R. G. Cooke, "Gibbs' phenomenon in Fourier-Bessel series and integrals", Proc. London Math. Soc. (2) 27 (1928), 171-192.

[Gibbs 1898] J. W. Gibbs, letters in Nature 59 (189899), pp. 200, 606. Reprinted as pp. 258-260 in Collected Works, vol. 2, Longmans, New York, 1927.

[Hewitt and Hewitt 1980] E. Hewitt and R. Hewitt, "The Gibbs-Wilbraham Phenomenon: An Episode in Fourier Analysis", Archives for the History of Exact Sciences 21 (1980), 129-160.

[Pinsky 1991] M. A. Pinsky, Partial Differential Equations and Boundary Value Problems with Applications, 2nd ed., McGraw-Hill, New York, 1991.

[Watson 1966] G. N. Watson, A Treatise on the Theory of Bessel Functions, Cambridge University Press, Cambridge (UK), 1966.

[Weyl 1909] H. Weyl, "Die Gibbssche Erscheinung in der Theorie der Kugelfunktionen", Rend. Circ. Math. Palermo 29 (1909), 308-323. Reprinted as pp. 305320 in Gesammelte Abhandlungen, vol. 1, SpringerVerlag, Berlin, 1968.

[Wilbraham 1848] H. Wilbraham, "On a certain periodic function", Cambridge and Dublin Math. J. 3 (1848), 198-201.

Alfred Gray, Department of Mathematics, University of Maryland, College Park, Maryland 20742 (gray@athena.umn.edu)

Mark A. Pinsky, Department of Mathematics, Northwestern University, Evanston, Illinois, 60208

(mpinsky@math.nwu.edu)

Received March 21, 1992; accepted in revised form January 30, 1993 